\section{Research Square}

\title{
MicroRNA-17-5p Downregulation Inhibits Autophagy and Myocardial Remodeling after Myocardial Infarction by Targeting STAT3
}

\section{Bo Chen}

First People's Hospital of Jiashan County

\section{Yinjun Yang}

First People's Hospital of Jiashan County

Jinbo Wu

Dongguan Hospital of Traditional chinese Medicine

Jianjiang Song

First People's Hospital of Jiashan County

Jia Lu ( $\nabla$ llujia0904@163.com )

First People's Hospital of Jiashan County

\section{Research Article}

Keywords: Myocardial infarction, Myocardial remodeling, microRNA-17-5p, STAT3, Autophagy, Myocardial fibrosis, Cardiomyocyte apoptosis

Posted Date: March 11th, 2021

DOI: https://doi.org/10.21203/rs.3.rs-272426/v1

License: (c) (1) This work is licensed under a Creative Commons Attribution 4.0 International License. Read Full License 


\section{Abstract}

Objective: Myocardial infarction (MI) is acknowledged as a major cause of heart disease related death in the world. This study aims to explore the function and mechanism of miR-17-5p-induced STAT3 in myocardial remodeling after Ml.

Methods: MI mice were infected by lentivirus antago-miR-17-5p vector. The expression of miR-17-5p in myocardial tissues was detected. The levels of STAT3 and specific markers of autophagy LC3-II/LC3-I, Beclin-1 and p62 were measured. Myocardial remodeling and scar size were assessed and cell apoptosis was determined. The binding of miR-17-5p and STAT3 was analyzed. The functions of miR-17-5p and STAT3 in autophagy and myocardial remodeling after MI were confirmed by functional rescue experiment after injecting STAT3 inhibitor S31-20.

Results: Decreased fractional shortening, increased left ventricular systolic diameter and ventricular filling velocity were detected in MI mice. High expression of miR-17-5p was found in myocardial tissues after MI. The inhibition of miR-17-5p expression led to reduction of fibrosis of myocardial tissues, scarring, cardiomyocyte apoptosis, and expressions of LC3-II/LC3-I and Beclin-1, and increased p62 expression after MI. miR-17-5p targeted STAT3 and negatively regulated its expression. Inhibition of STAT3 level reversed the improving effect of antago-miR-17-5p on MI.

Conclusion: Inhibition of miR-17-5p can inhibit myocardial autophagy through targeting STAT3, and then inhibit myocardial remodeling, thereby protecting myocardium after $\mathrm{Ml}$.

\section{Introduction}

Myocardial infarction (MI) appears when the duration of ischemia is sufficient to bring out myocyte necrosis [1]. Ml is accompanied by inflammatory responses which lead to myocardial remodeling, manifesting as cardiac dilatation, poor cardiac function, cardiac arrhythmias, so far as to heart failure [2, 3]. The remodeling processes comprise a range of stages of interdependence, comprising inflammatory conditions, cell multiplication and repair [4]. This remodeling significantly influences the ventricle performance and the survival prognosis in MI [5]. As a primary cellular process, autophagy is related to the degradation and recovery of the non-essential and dysfunctional proteins, organelles, and other related cellular components [6]. Chronic cardiac remodeling in mouse models of myocardial ischemia and MI was exacerbated due to genetic or pharmacological inhibition of autophagy [7]. As is known to all, the intersection for post-infarction injury lies in cardiomyocyte apoptosis, resulting in ventricular remodeling and cardiac failure [8]. Therefore, more studies are needed to probe the specific mechanism involved in these processes.

MicroRNAs (miRNA) are endogenous, conserved 19-25 non-coding RNAs [9]. Recent study has demonstrated that pathogenic changes in tissues, including cardiac hypertrophy, heart failure, and cardiac fibrosis, may be associated with miR [10]. miRNAs take control of various biological processes, including cell proliferation, apoptosis and autophagy [11]. For instance, as a member of miR-17-92 
cluster, miR-17-5p is modulated by vascular endothelial growth factor, and may participate in neoangiogenesis, promoting blood vessel growth and functional restoration of ischemic tissue [12]. Recent study has shown that miR-17-5p is a responsive miRNA of hypoxia-induced autophagy and apoptosis in vascular smooth muscle cells via targeting STAT3 [10]. STAT3 can offer cardiac protection, which also is associated with the modification of miRNA expression profiles in cardiomyocytes [13]. The 3'UTR of the STAT3 gene with the predicated target sites of miR-17-5p was detected utilizing several miRNA databases in a previous research [14]. One previous work has shown that different localization of STAT3 may affect autophagy differently [15]. Researches have been investigated the biological significance of STAT3 activity in sub-acute MI $[16,17]$. However, there is no report on the effect of the miR-17-5p/STAT3 axis on autophagy and myocardial remodeling after $\mathrm{Ml}$ at home and abroad.

In this study, we investigated the protective mechanism of miR-17-5p/STAT3 pathway in myocardial remodeling after $\mathrm{Ml}$ by affecting autophagy. We hope to provide novel insight into the management of myocardial remodeling after MI.

\section{Material And Methods}

\section{Establishment and grouping of MI mice}

The MI mouse model was established in accordance with a previous study [18] and specific methods were illustrated as follows. Healthy adult C57BL/ 6 mice of the same age were purchased from Beijing Beike Huaxia Biomedical Technology Co., Ltd. [SYXK (Beijing) 2017-0044, Beijing, China]. They were raised under the condition of temperature at $20-25^{\circ} \mathrm{C}$ with day and night cycle and water and food were taken freely. Mice were allowed $72 \mathrm{~h}$ to accommodate to the environment before the experiment. Then, mice were anesthetized using pentobarbital sodium (i.p., $60 \mathrm{mg} / \mathrm{kg}$ ), and then mouse limbs were attached to the electrocardiographic (ECG) monitoring electrode (BeneHearth R3; Mindray, Wuhan, Hubei, China). Subsequently, mice were orally attached to the small-animal ventilators (R407, Rayto Life Science, Shenzhen, China) with indoor air as the source, the breathing frequency of $60 \mathrm{times} / \mathrm{min}$, and the tidal volume of $13-15 \mathrm{~mL} / \mathrm{kg}$. A skin incision was made in the fourth left intercostal space to expose the heart and ligated using $6 / 0$ atraumatic suture through anterior descending coronary artery. Obvious elevation of ST-segment was revealed by the ECG monitoring, which meant the success of vascular occlusion. After 60 min of occlusion, the arterial coronary was reopened by releasing the suture. The ECG monitoring revealed that the ST-segment was recovered, indicating the successful establishment of mouse models of MI. The same operation was conducted to the sham group, but the sutures were crossed through under left anterior descending (LAD) without ligation.

The modeled mice were assigned into 6 groups with 24 in each group: sham group, MI group (MI operation group), $\mathrm{Ml}+$ antago-NC group (mice were injected with antago-NC after Ml operation), $\mathrm{Ml}+$ antago-miR-17-5p group (mice were injected with antago-miR-17-5p after Ml operation), MI + S31-201 (mice were injected with S31-201 after MI operation), MI + S31-201 + antago-miR-17-5p group (mice were injected with antago-miR-17-5p on the 1st day after MI operation and STAT3 inhibitor S31-201 on the 7th 
day after MI operation). The 6 mice in each group were used for TUNEL and for Masson staining, 6 mice for Western blot analysis, 6 mice for 2,3,5-Triphenyltetrazolium chloride (TTC) staining and 6 mice for cerebral edema. The antago-NC and antago-miR-17-5p were purchased from Syngen (Beijing, China) and S31-201 (a STAT3 inhibitor) was from Calbiochem, La Jolla, CA, USA [19]. The $25 \mu \mathrm{L}$ antagomiRNA was injected into myocardial tissue around the ligation site of the heart at 3 points. The first injection was performed on the 1st day to eliminate the protective effects to acute MI. Then intravenous injection was performed every 3 days until the 28th day after MI operation. On the 28th day after operation, the mice were euthanized using intravenous or intraperitoneal injection of 3 times the amount of anesthetic barbiturates after ECG monitoring.

\section{Transthoracic echocardiography (TTE)}

On the 28th day after the operation, mice in each group were anesthetized by an intraperitoneal injection of pentobarbital sodium ( $60 \mathrm{mg} / \mathrm{kg}$ ) and were checked by TTE using Color Doppler-type ultrasonic diagnostic apparatus (Sequoia 512; Acuson, Mountain View, CA, USA) at $8.5 \mathrm{~m} / \mathrm{Hz}$ and $100 \mathrm{~mm} / \mathrm{s}$. The anesthetized mice were fixed on the testing platform. The fractional shortening (FS) of short axis, left ventricular internal dimention in systole (LVIDs), and ventricular diastolic filling velocity (E-wave velocity: A-wave velocity, E/A ratio) were continuously measured and averaged. The cardiac function of mice and its correlation with the above indexes were analyzed.

\section{TTC staining}

TTC staining method was as previously mentioned [18]. Following TTE, the hearts were taken out from the mice and frozen for half an $\mathrm{h}$ at $-80^{\circ} \mathrm{C}$, and then the transverse section across the left ventricular long axis from the apex to the bottom was obtained using a vibrating blade. The section was added with $1 \%$ TTC for staining after weighing on the electronic balance. The infarcted myocardium was gray and the viable myocardium was brick red. These two types of myocardium were separated and then each was weighed on the electronic balance after drying the dye liquor with filter paper. The myocardial infarction size $=$ weight of infarcted myocardium/total weight of left ventricular myocardium $\times 100 \%$.

\section{Transmission electron microscope (TEM)}

Specimens were prepared according to the conventional specimen preparation technology of TEM. The myocardial tissues at the location needed by the experiment were cut and trimmed to $1 \mathrm{~mm}^{3}$ pieces and then fixed in $4 \%$ paraformaldehyde solution at $4^{\circ} \mathrm{C}$. After being fully washed by $0.1 \mathrm{~mol} / \mathrm{L}$ phosphate buffered saline (PBS), the sections were fixed at $1 \%$ osmium tetroxide for $2 \mathrm{~h}$, followed by conventional dehydration by gradient ethanol or acetone, and soaking and embedding in ethoxyline resin. Ultrathin sections were prepared and stained using uranyl acetate and lead citrate, and observed and photographed under the scanning electron microscope.

\section{Masson staining}

After cardiac perfusion, the specimens were fixed with $10 \%$ buffered formalin, cut into pieces at $5 \mathrm{~mm}$, stained using Weigert iron hematoxylin, and washed in running water. The sections were then acidized by 
acidic ethanol for a few seconds, washed for several minutes and stained with blue dye solution for a few seconds. Then the sections were washed for several minutes, and stained with Ponceau fuchsin staining solution for several minutes. Acetic acid working solution was prepared with distilled water and acetic acid solution in certain proportion and then used to wash the section. After phosphomolybdic acid treatment, the sections were counterstained with aniline blue dye solution, and washed by acetic acid working solution until no blue color. Then the sections were dehydrated with ethanol immediately and mounted with neutral resins.

\section{Reverse transcription-quantitative polymerase chain reaction (RT-qPCR)}

RT-qPCR was performed as mentioned in the reference [18].The TRIzol one-step method was employed to extract total RNA of myocardial tissues based on the instructions of TRIzol reagent (Invitrogen Inc., Carlsbad, CA). RNA was dissolved in ultrapure water treated by diethyl pyrocarbonate. Absorbances at the wavelengths of $260 \mathrm{~nm}$ and $280 \mathrm{~nm}$ were detected using ultraviolet-visible spectrophotometer (ND-1000, NanoDrop Technologies, Wilmington, DE, USA). The quality of total RNA was confirmed and total RNA concentration was adjusted. The experiment conditions were: constant temperature at $70^{\circ} \mathrm{C}$ for $10 \mathrm{~min}$, ice-bath for $2 \mathrm{~min}$, constant temperature at $42^{\circ} \mathrm{C}$ for $60 \mathrm{~min}$, and at $70^{\circ} \mathrm{C}$ for $10 \mathrm{~min}$. The complementary DNA obtained from reverse transcription was stored at $-80^{\circ} \mathrm{C}$ temporarily. The reaction system was operated by TaqMan probe method in line with the kit (Fermentas Inc., Vilnius, Lithuania) instructions on $A B I 7500$ instrument ( $A B I$, Foster City, $C A$, USA) with U6 as the internal control. The expression was determined with $2^{-\triangle \triangle C T}$ method. The primer sequences are illustrated in Table 1.

Table 1

Primer sequences

\begin{tabular}{|ll|}
\hline Name of primer & Sequences \\
\hline miR-17-5p forward & 5'-TCTAGATCCCGAGGACTG-3' $^{\prime}$ \\
\hline miR-17-5p reverse & 5'-ATCGTGACCTGAACC-3' \\
\hline U6 forward & 5'-CTCGCTTCGGCAGCACA-3' \\
\hline U6 reverse & 5'-AACGCTTCACGAATTTGCGT-3' \\
\hline
\end{tabular}

\section{Western blot}

RIPA lysate was used and heart tissues were centrifuged for $20 \mathrm{~min}$ at $8000 \mathrm{xg}$. Protein concentration in supernatant was determined using a Bradford kit (Pierce Biotechnology, Rockford, IL, USA). Protein samples $(30 \mu \mathrm{g})$ were segregated with $10 \%$ sodium dodecyl sulfate-polyacrylamide gel electrophoresis and transferred onto cellulose nitrate membranes (Bio-Rad Laboratories, Hercules, CA, USA). The membranes were blocked using $5 \%(\mathrm{w} / \mathrm{v})$ nonfat dry milk and $0.1 \%(\mathrm{w} / \mathrm{v})$ Tween $20($ TBST) for $2 \mathrm{~h}$, and then the membranes were incubated with light chain 3 (LC3, sc-292,354, 1:1,000), p62 (sc-48389, 1:1,000), $\beta$-actin (sc-81178, 1:2,000), Beclin-1 (ab207612, 1:1,000), STAT3 (sc-483, 1:1,000) at $4^{\circ} \mathrm{C}$ overnight. The 
membranes were washed in TBST 3 times and incubated with the secondary antibody donkey anti-rabbit IgG (sc-2096, 1:10) and goat anti-rabbit IgG (sc-2004, 1:10) at room temperature for $2 \mathrm{~h}$. Enhanced chemiluminescence was used to expose protein band and relative protein was determined by ImageJ software (the National Institutes of Health, Bethesda, MD, USA). All antibodies were purchased from Santa Cruz Biotechnology Inc. (Dallas, TX, USA).

\section{Dual-luciferase reporter assay}

Luciferase plasmid was constructed as follows: the wild-type (wt) sequence containing the target gene STAT3-3'-UTR fragment was constructed and was complementary to corresponding miR-17-5p. Then, the wt sequence fragment of STAT3 3'-UTR-wt containing predicted binding site of miR-17-5p and the mutant (mut) sequence fragment of STAT3 3'-UTR-mut were cloned to pGL3-basic vector. The 293T cells (Chinese Academy of Sciences, GNHu17, Shanghai, China) were evenly seeded in 6-well culture plate at the concentration of $3 \times 10^{5} / \mathrm{mL}, 1 \mathrm{~mL}$ for each well. Subsequently, the 293T cells were co-transferred with luciferase plasmid containing STAT3 3'-UTR-wt or STAT3 3'-UTR-mut and miR-17-5p mimic or mimic control. A normal control group was set. Cells were harvested $48 \mathrm{~h}$ post-transfection and measured with luciferase reporter assay kit. The absorbance at the wavelength of $560 \mathrm{~mm}$ was detected by a microplate reader.

\section{TUNEL}

DeadEndTM luciferase TUNEL system (Promega, Madison, WI, USA) was applied to detect cardiomyocyte apoptosis. The fixed heat tissue slices were permeabilized in PBS containing $0.2 \%$ Triton X-100 for 5 min, and cut and equilibrated for $10 \mathrm{~min}$ at room temperature, and then incubated with terminal deoxynucleotidyl transferase (TDT) reaction mixture for $60 \mathrm{~min}$ in a humidified chamber at $37^{\circ} \mathrm{C}$. Finally, the nuclei were labeled by Hoechst 33258 (Invitrogen). Apoptotic cells were observed under a fluorescence microscopy. Green florescence was observed in the apoptotic cells with blue nuclei. At least 6 visual fields were randomly selected to calculate the percentage of apoptosis.

\section{Statistical analysis}

Statistical analyses were performed using SPSS 21.0 (IBM Corp. Armonk, NY, USA). Kolmogorov-Smirnov was used to analyze data were in normal distribution. Data are expressed as the mean \pm standard deviation (SD). Comparisons between 2 groups were performed using $t$-test and comparisons between multiple groups were performed using one-way analysis of variance (ANOVA) or two-way ANOVA, followed by Tukey's multiple comparisons test. $P$ value was obtained using a two-sided test. $P<0.05$ was considered a statistically significant difference.

\section{Results}

\section{miR-17-5p was highly expressed in myocardial tissue after MI}


Cardiac function parameters of mice in $\mathrm{Ml}$ and sham groups were detected by electrocardiogram. FS was reduced (Fig. 1A), LVIDs was elevated (Fig. 1B), and E/A filling velocity was elevated (Fig. 1C), indicating that mice developed serious systolic and diastolic dysfunction after MI operation. Several studies have shown that miR may be related to histopathological changes of myocardial hypertrophy, heart failure, myocardial fibrosis and vascular atherosclerosis $[9,20]$. miR-17-5p promoted the apoptosis of cells induced by oxidative stress in animal models of myocardial injury induced by ischemia reperfusion and cell models of cardiomyocyte injury [14]. The expression of miR-17-5p in myocardial tissues was examined at 28 days after $\mathrm{Ml}$ and the result showed that miR-17-5p expression was significantly increased $(P<0.05$, Fig. 1D). Above results indicated that miR-17-5p could be related to Ml.

\section{Inhibition of miR-17-5p improved ventricular remodeling and reduced myocardial apoptosis after $\mathrm{MI}$}

To explore the function of miR-17-5p in myocardial reconstruction after Ml, antago-miR-17-5p was injected into the myocardial tissues. Lowered expression of miR-17-5p was detected by RT-qPCR (Fig. 2A), and cardiac function of mice in each group was examined by ECG. Compared with mice in MI group, the cardiac function was remarkably improved after the inhibition of miR-17-5p (Fig. 2B-D). The infarcted area was measured by TTC staining. Compared with mice in MI group, the infarcted area was reduced after the inhibition of miR-17-5p (all $P<0.05$, Fig. 2E). Masson staining showed that the fibrosis and scar formation (blue staining) of myocardial tissue in $\mathrm{MI}$ mice were increased, but were decreased after the inhibition of miR-17-5p (Fig. 2F). Similarly, TUNEL staining showed that apoptotic rate of myocardial tissues was increased after Ml but decreased after the inhibition of miR-17-5p $(P<0.05$, Fig. $2 G)$. All the results indicated that the inhibition of miR-17-5p improved the remodeling and myocardial apoptosis after MI.

\section{Inhibition of miR-17-5p suppressed autophagy in cardiomyocyte}

To explore the underlying mechanism of the inhibition of miR-17-5p in remodeling and myocardial apoptosis after $\mathrm{Ml}$, the effect of miR-17-5p on autophagy was analyzed. The sarcomere structure of hypertrophic myocardial tissue and mitochondria array of cardiomyocyte in antago-miR-17-5p group were observed using transmission electron microscopy. Compared with the control group, the typical autophagic vacuole in degraded organelles, including mitochondria and endoplasmic reticulum, were reduced after inhibiting miR-17-5p expression (Fig. 3A). Western blot result showed that after inhibiting miR-17-5p expression, LC3-II/LC3-I and Beclin-1 in the myocardial tissues were decreased while the expression of p62 was increased significantly (Fig. 3B). These results demonstrated that the inhibition of miR-17-5p suppressed the autophagy in cardiomyocyte.

\section{miR-17-5p targeted STAT3}

To find out the potential mechanism of miR-17-5p regulating autophagy after $\mathrm{MI}$, we predicted the target gene of miR-17-5p using database Starbase online, and STAT3 caught our attention (Fig. 4A). STAT3 
plays a key role in myocardial protection [21]. The binding relation between miR-17-5p and STAT3 was further identified by a dual-luciferase assay. The transfection of miR-17-5p mimic greatly reduced the luciferase activity of STAT3-wt plasmid in STAT3 3'-UTR-wt group $(P<0.01$, Fig. 4B). It indicated that miR17-5p could act directly on the predicted target sites of STAT3 3'-UTR. Western blot showed that the level of STAT3 protein in antago-miR-17-5p group was higher than $\mathrm{Ml}$ and antago-NC groups $(P<0.01, \mathrm{Fig} .4 \mathrm{C})$, suggesting that inhibition of miR-17-5p upregulated the levels of STAT3 protein. The results above demonstrated that miR-17-5p negatively regulates the levels of STAT3 protein.

\section{Inhibition of STAT3 reversed the improving effect of antago-miR-17-5p on Ml}

To investigate whether STAT3 is involved in the effect of miR-17-5p on myocardial reconstruction after MI, we injected STAT3 inhibitor S31-20 into the myocardial tissues around the ligation site of mouse hearts. The expressions of specific markers of autophagy LC3, Beclin-1 and p62 were detected using Western blot, and the result showed increased LC3-II/LC3-I and Beclin-1 expression and decreased p62 in myocardial tissues after inhibiting STAT3 and miR-17-5p expression compared with antago-miR-17-5p group (all $P<0.01$, Fig. 5A). This result indicated that the inhibition of STAT3 promoted autophagy in myocardial tissues and reversed the inhibition of antago-miR-17-5p on autophagy. According to the infarction size measured by TTC staining, the inhibition of both STAT3 and miR-17-5p expression blocked the effect of antago-miR-17-5p on infarction size ( $P<0.01$, Fig. 5B). Masson staining showed that fibrosis and scar formation in myocardial tissues were decreased after inhibiting miR-17-5p expression, and the condition was reversed after STAT3 expression was inhibited ( $P<0.01$, Fig. 5C). TUNEL staining showed that myocardial apoptosis was reduced after inhibiting miR-17-5p expression, while the inhibition of STAT3 not only increased apoptosis, but also blocked the protective effect of antago-miR-17-5p on MI ( $P$ $<0.01$, Fig. 5D). In summary, the inhibition of STAT3 reversed the improving effect of antago-miR-17-5p on $\mathrm{MI}$.

\section{Discussion}

Myocardial infarction is a major cause of death and disability worldwide, which is followed by remodeling of the surrounding myocardium [22]. The process of remodeling includes hypertrophy and fibrosis of the left ventricular wall, and this could lead to impaired cardiac function and even death [23]. Autophagy has been reported to act as an inflammatory suppressor and contribute to the alleviation of progress of cardiac injury [24]. In present study, we investigated the role of miR-17-5p/STAT3 on myocardial remodeling in MI mice through autophagy. And the results showed that miR-17-5p/STAT3 repressed autophagy and inhibited myocardial remodeling after MI.

According to the results, high expression of miR-17-5p was detected in myocardial tissues after Ml, which was consistent to the findings of another study that significant upregulation of miR-17-5p expression was observed in mouse myocardium with ischemia/reperfusion-induced cardiac injury [14]. Excessive myocardial fibrosis is the main pathological process in the development of myocardial remodeling and 
thus preventing excessive myocardial fibrosis is important [25]. Apoptosis also has been suggested to be responsible for a significant amount of cardiomyocyte death [26]. In this study, we inhibited the expression of miR-17-5p using antago-miR-17-5p, and then noticed that the infarcted area, fibrosis and apoptotic rate of myocardial tissue were decreased. Zhao et al. have revealed that inhibition of miR-17-5p leads to decreased apoptosis related to endoplasmic reticulum stress response in the hypoxia model [27]. Another research also has demonstrated that miR-17-5p silencing has a great apoptosis inhibition effect on endothelial cells under serum-deprived conditions [28]. Therefore, the results demonstrated that the inhibition of miR-17-5p expression improved the condition of remodeling and myocardial apoptosis after MI.

Autophagy can act as an inflammatory suppressor, thus contributing to the alleviation of progress of cardiac injury [24]. It has been studied that miR-17-5p mediates hypoxia-induced autophagy and inhibits apoptosis by STAT3 in vascular smooth muscle cells [10]. In our study, the typical autophagic vacuole in degraded organelles, including mitochondria and endoplasmic reticulum, were reduced after inhibiting miR-17-5p expression, and LC3-II/LC3-I and Beclin-1 in the myocardial tissues were decreased while p62 was increased significantly. Studies have found that miR-17-5p regulates autophagy to promote hepatic ischemia/reperfusion injury via suppression of STAT3 expression [29-31]. In conclusion, our study suggested that the inhibition of miR-17-5p suppressed cardiomyocyte autophagy.

STAT3 is reported to be inhibited by miR-17-5p to promote apoptosis in breast cancer cells [32]. In this study, the binding of miR-17-5p and STAT3 was proved by the dual-luciferase assay. The levels of STAT3 protein in antago-miR-17-5p mice were higher than MI mice and mice in antago-NC control group. miR-17$5 p$ could target the STAT3 3'UTR to inhibit the expression of STAT3 in myeloid-derived suppressor cells [33]. Consequently, the results above indicated that miR-17-5p targeted STAT3 in myocardial tissues. STAT3, as a key cellular survival factor provides cardioprotection from ischemic reperfusion injury, so activation of STAT3 in the stressed heart is important for its protection of cardiomyocytes from specific apoptotic stimuli [16]. In our study, we injected STAT3 inhibitor S31-20 into MI mice treated with antagomiR-17-5p and then found the improved condition of fibrosis and apoptosis was deteriorated again. Excessive autophagy could aggravate the myocardial ischemia-reperfusion injury [34]. STAT3 acts as a repressor of autophagy and has anti-fibrotic effects during cardiac remodeling [17, 35]. Blocking STAT3 phosphorylation by chemical inhibitor AG490 induced increases in Atg3 and LC3-II/LC3-I in high glucosestimulated RSC96 cells [36]. Knockdown of STAT3 synchronously suppressed the fibrogenesis and autophagic flux [37]. Thus, the result demonstrated that the inhibition of STAT3 expression reversed the improving effect of antago-miR-17-5p to MI mice.

In summary, this study has found that miR-17-5p/STAT3 inhibited myocardial remodeling post-MI by blocking autophagy pathway. However, this study only revealed that the inhibition of miR-17-5p expression and upregulation of STAT expression inhibited autophagy, and further inhibited myocardial remodeling post-MI and then to some extent sped the healing of myocardium post-MI. But the mechanism of STAT3 is not explored further enough. Future studies should pay attention to the potential functional mechanism of STAT3 in myocardial remodeling post-MI. 


\section{Declarations}

\section{Acknowledgements}

Not applicable.

\section{Authors' Contributions}

B.C. and Y.Y. contributed to the study concepts, study design, and definition of intellectual content; B.C., Y.Y. and J.W. contributed to the literature research; B.C. and Y.Y. contributed to the manuscript preparation; J.L. contributed to the manuscript editing and review; B.C., J.W. and J.S. contributed to the experimental studies and data acquisition; J.L., Y.Y., J.W. and J.S. contributed to the data analysis and statistical analysis. All authors read and approved the final manuscript.

\section{Funding}

Not applicable.

\section{Availability of data and materials}

The data that support this study are available from the corresponding author upon reasonable request.

\section{Ethics approval and consent to participate}

This study was approved by Ethics Committees of the First People's Hospital of Jiashan County. This study was in accordance with national animal protection and welfare, as well as ethical principles. We had done our utmost to cut the animal amount and sufferings. All methods are reported in accordance with ARRIVE guidelines.

\section{Consent for publication}

Not applicable.

\section{Competing interests}

The authors declare that they have no competing interests.

\section{References}


1. Mouton AJ, Rivera OJ, Lindsey ML. Myocardial infarction remodeling that progresses to heart failure: a signaling misunderstanding. Am J Physiol Heart Circ Physiol 2018; 315(1):H71-H9.

2. Takahashi M. Role of NLRP3 Inflammasome in Cardiac Inflammation and Remodeling after Myocardial Infarction. Biol Pharm Bull 2019; 42(4):518-23.

3. Ren PH, Zhang ZM, Wang P, Zhu HP, Li ZQ. Yangxinkang tablet protects against cardiac dysfunction and remodelling after myocardial infarction in rats through inhibition of AMPK/mTOR-mediated autophagy. Pharm Biol 2020; 58(1):321-7.

4. Sanchez-Alonso S, Alcaraz-Serna A, Sanchez-Madrid F, Alfranca A. Extracellular Vesicle-Mediated Immune Regulation of Tissue Remodeling and Angiogenesis After Myocardial Infarction. Front Immunol 2018; 9:2799.

5. Pfeffer MA, Braunwald E. Ventricular remodeling after myocardial infarction. Experimental observations and clinical implications. Circulation 1990; 81(4):1161-72.

6. Wang X, Guo Z, Ding Z, Mehta JL. Inflammation, Autophagy, and Apoptosis After Myocardial Infarction. J Am Heart Assoc 2018; 7(9).

7. Sciarretta S, Yee D, Nagarajan N, Bianchi F, Saito T, Valenti V, et al. Trehalose-Induced Activation of Autophagy Improves Cardiac Remodeling After Myocardial Infarction. J Am Coll Cardiol 2018; 71(18):1999-2010.

8. Li X, Zhao J, Geng J, Chen F, Wei Z, Liu C, et al. Long non-coding RNA MEG3 knockdown attenuates endoplasmic reticulum stress-mediated apoptosis by targeting p53 following myocardial infarction. J Cell Mol Med 2019; 23(12):8369-80.

9. Pan ZW, Lu YJ, Yang BF. MicroRNAs: a novel class of potential therapeutic targets for cardiovascular diseases. Acta Pharmacol Sin 2010; 31(1):1-9.

10. Hao MX, Wang X, Jiao KL. MicroRNA-17-5p mediates hypoxia-induced autophagy and inhibits apoptosis by targeting signal transducer and activator of transcription 3 in vascular smooth muscle cells. Exp Ther Med 2017; 13(3):935-41.

11. Akbari G. Emerging roles of microRNAs in intestinal ischemia/reperfusion-induced injury: a review. J Physiol Biochem 2020; 76(4):525-37.

12. Xue S, Liu D, Zhu W, Su Z, Zhang L, Zhou C, et al. Circulating MiR-17-5p, MiR-126-5p and MiR-145-3p Are Novel Biomarkers for Diagnosis of Acute Myocardial Infarction. Front Physiol 2019; 10:123.

13. Harhous Z, Booz GW, Ovize M, Bidaux G, Kurdi M. An Update on the Multifaceted Roles of STAT3 in the Heart. Front Cardiovasc Med 2019; 6:150.

14. Du W, Pan Z, Chen $X$, Wang $L$, Zhang $Y$, Li S, et al. By targeting Stat3 microRNA-17-5p promotes cardiomyocyte apoptosis in response to ischemia followed by reperfusion. Cell Physiol Biochem 2014; 34(3):955-65.

15. Li H, Chen L, Li JJ, Zhou Q, Huang A, Liu WW, et al. miR-519a enhances chemosensitivity and promotes autophagy in glioblastoma by targeting STAT3/Bcl2 signaling pathway. J Hematol Oncol 2018; 11(1):70. 
16. He F, Liu H, Guo J, Yang D, Yu Y, Yu J, et al. Inhibition of MicroRNA-124 Reduces Cardiomyocyte Apoptosis Following Myocardial Infarction via Targeting STAT3. Cell Physiol Biochem 2018; 51(1):186-200.

17. Enomoto D, Obana M, Miyawaki A, Maeda M, Nakayama H, Fujio Y. Cardiac-specific ablation of the STAT3 gene in the subacute phase of myocardial infarction exacerbated cardiac remodeling. Am J Physiol Heart Circ Physiol 2015; 309(3):H471-80.

18. Liu ZY, Pan HW, Cao Y, Zheng J, Zhang Y, Tang Y, et al. Downregulated microRNA-330 suppresses left ventricular remodeling via the TGF-beta1/Smad3 signaling pathway by targeting SRY in mice with myocardial ischemia-reperfusion injury. J Cell Physiol 2019; 234(7):11440-50.

19. Lin CC, Chen SY, Lien HY, Lin SZ, Lee TM. Targeting the PI3K/STAT3 axis modulates age-related differences in macrophage phenotype in rats with myocardial infarction. J Cell Mol Med 2019; 23(9):6378-92.

20. Urbich C, Kuehbacher A, Dimmeler S. Role of microRNAs in vascular diseases, inflammation, and angiogenesis. Cardiovasc Res 2008; 79(4):581-8.

21. Yang Y, Hu W, Di S, Ma Z, Fan C, Wang D, et al. Tackling myocardial ischemic injury: the signal transducer and activator of transcription 3 (STAT3) at a good site. Expert Opin Ther Targets 2017; 21(2):215-28.

22. Thygesen K, Alpert JS, White HD, Joint ESCAAHAWHFTFftRoMI. Universal definition of myocardial infarction. J Am Coll Cardiol 2007; 50(22):2173-95.

23. Talman $\mathrm{V}$, Ruskoaho $\mathrm{H}$. Cardiac fibrosis in myocardial infarction-from repair and remodeling to regeneration. Cell Tissue Res 2016; 365(3):563-81.

24. Wu D, Zhang K, Hu P. The Role of Autophagy in Acute Myocardial Infarction. Front Pharmacol 2019; 10:551.

25. Yuan J, Liu H, Gao W, Zhang L, Ye Y, Yuan L, et al. MicroRNA-378 suppresses myocardial fibrosis through a paracrine mechanism at the early stage of cardiac hypertrophy following mechanical stress. Theranostics 2018; 8(9):2565-82.

26. Takemura G, Fujiwara H. Role of apoptosis in remodeling after myocardial infarction. Pharmacol Ther 2004; 104(1):1-16.

27. Zhao L, Jiang S, Wu N, Shi E, Yang L, Li Q. MiR-17-5p-mediated endoplasmic reticulum stress promotes acute myocardial ischemia injury through targeting Tsg101. Cell Stress Chaperones 2020.

28. Yang S, Fan T, Hu Q, Xu W, Yang J, Xu C, et al. Downregulation of microRNA-17-5p improves cardiac function after myocardial infarction via attenuation of apoptosis in endothelial cells. Mol Genet Genomics 2018; 293(4):883-94.

29. Yang J, Shi G, Gong Y, Cai J, Zheng Y, Zhang Z. LncRNA 0003250 accelerates heart autophagy and binds to miR-17-5p as a competitive endogenous RNA in chicken induced by selenium deficiency. $J$ Cell Physiol 2021; 236(1):157-77.

30. Wang X, Chen J, Huang X. Rosuvastatin Attenuates Myocardial Ischemia-Reperfusion Injury via Upregulating miR-17-3p-Mediated Autophagy. Cell Reprogram 2019; 21(6):323-30. 
31. Li S, Zhang J, Wang Z, Wang T, Yu Y, He J, et al. MicroRNA-17 regulates autophagy to promote hepatic ischemia/reperfusion injury via suppression of signal transductions and activation of transcription-3 expression. Liver Transpl 2016; 22(12):1697-709.

32. Liao XH, Xiang Y, Yu CX, Li JP, Li H, Nie Q, et al. STAT3 is required for MiR-17-5p-mediated sensitization to chemotherapy-induced apoptosis in breast cancer cells. Oncotarget 2017; 8(9):15763-74.

33. Zhang M, Liu Q, Mi S, Liang X, Zhang Z, Su X, et al. Both miR-17-5p and miR-20a alleviate suppressive potential of myeloid-derived suppressor cells by modulating STAT3 expression. J Immunol 2011; 186(8):4716-24.

34. Zhao R, Xie E, Yang X, Gong B. Alliin alleviates myocardial ischemia-reperfusion injury by promoting autophagy. Biochem Biophys Res Commun 2019; 512(2):236-43.

35. Zhang YG, Zhu X, Lu R, Messer JS, Xia Y, Chang EB, et al. Intestinal epithelial HMGB1 inhibits bacterial infection via STAT3 regulation of autophagy. Autophagy 2019; 15(11):1935-53.

36. Du W, Wang N, Li F, Jia K, An J, Liu Y, et al. STAT3 phosphorylation mediates high glucose-impaired cell autophagy in an HDAC1-dependent and -independent manner in Schwann cells of diabetic peripheral neuropathy. FASEB J 2019; 33(7):8008-21.

37. Yuan Y, Zhang Y, Han X, Li Y, Zhao X, Sheng L, et al. Relaxin alleviates TGFbeta1-induced cardiac fibrosis via inhibition of Stat3-dependent autophagy. Biochem Biophys Res Commun 2017; 493(4):1601-7.

\section{Figures}
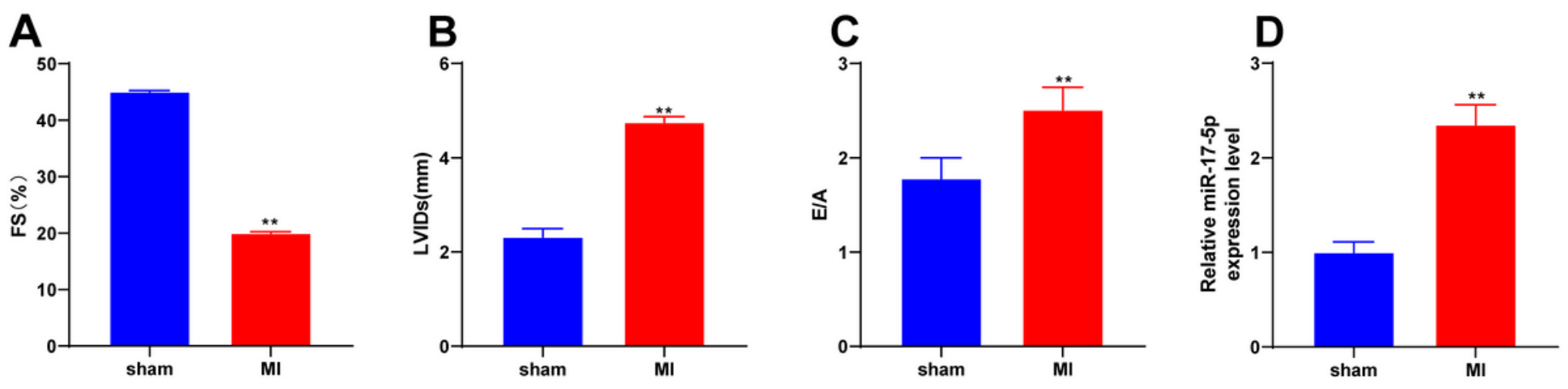

Figure 1

miR-17-5p was highly expressed in myocardial tissue after MI. Cardiac function parameters of mice were evaluated by ECG 28 days after operation. A: fractional shortening; B: left ventricular internal dimention in systole; C: ventricular diastolic filling velocity (E/A); D: the expression of miR-17-5p in myocardial tissues after MI detected by RT-qPCR. $\mathrm{N}=6$. The data are all measurement data, presented as mean \pm standard deviation, and analyzed using t-test. ${ }^{* *} \mathrm{P}<0.01$. 

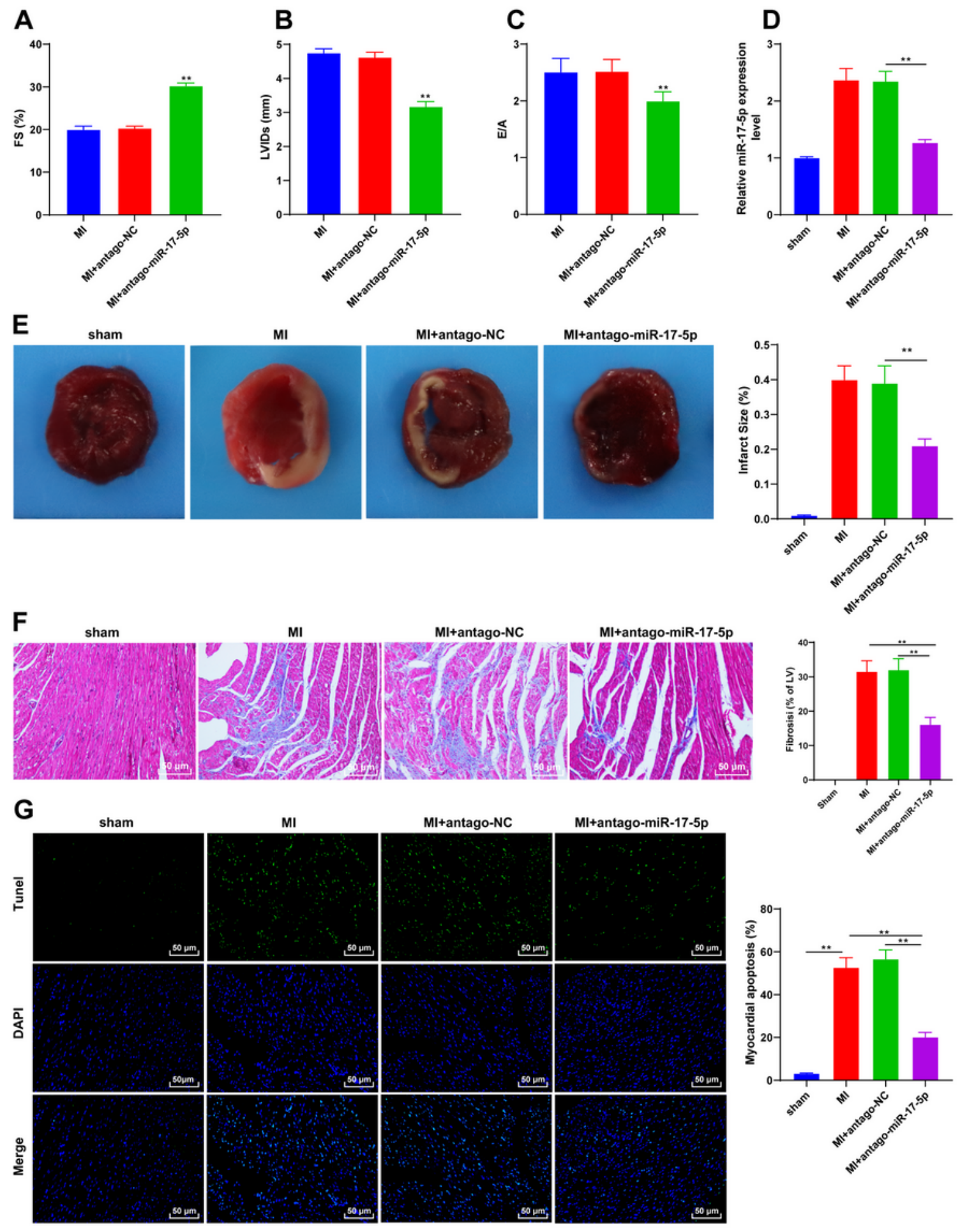

Figure 2

Inhibition of miR-17-5p expression improved ventricular remodeling and reduced myocardial apoptosis after MI. Antago-miR-17-5p was injected into the myocardial tissues, with antago-NC as the control. Cardiac function parameters of mice were evaluated by ECG 28 days after operation. (A) fraction shortening; (B) left ventricular internal dimention in systole; (C) ventricular diastolic filling velocity (E/A). Then (D) transfection efficiency of miR-17-5p was verified by RT-qPCR and (E) infarction size was 
measured by TTC staining method; (F) Masson staining was used to determine myocardial fibrosis after $\mathrm{Ml}$ (the tissues stained with blue color represented fibrosis) and the percentage of fibrosis area was compared among groups; (G) staining using TUNEL assay was performed to determine cell apoptosis. N $=6$. The data in the figure are all measurement data, presented as mean \pm standard deviation. Data were analyzed using one-way ANOVA, followed by Tukey's multiple comparisons test. ** $\mathrm{P}<0.01$.

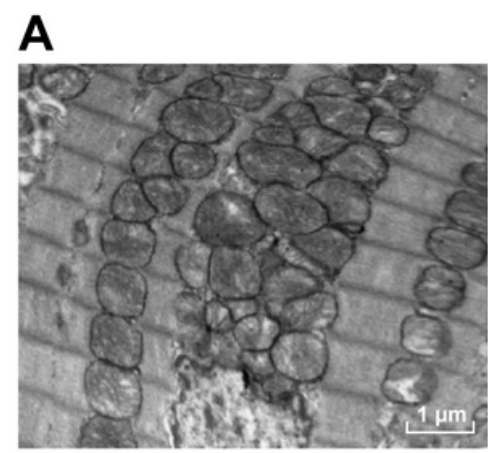

Sham

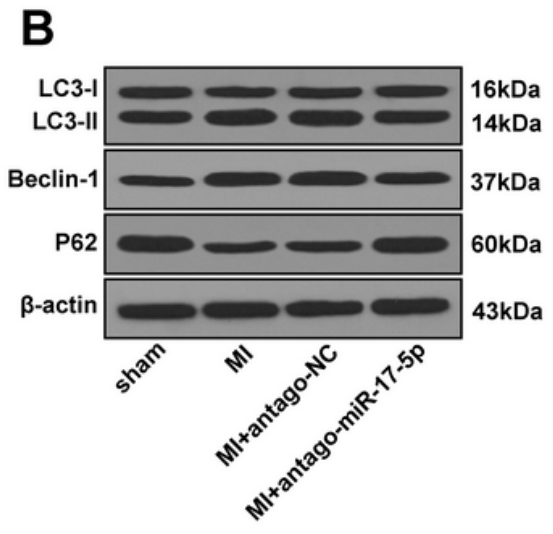

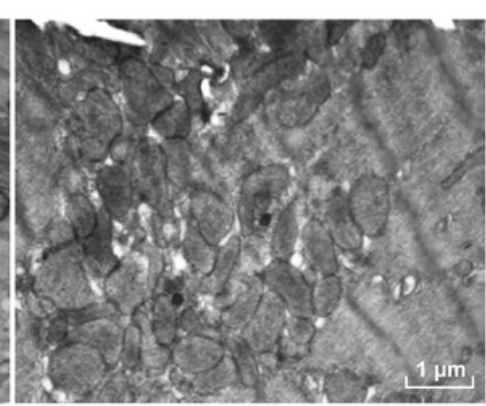

MI

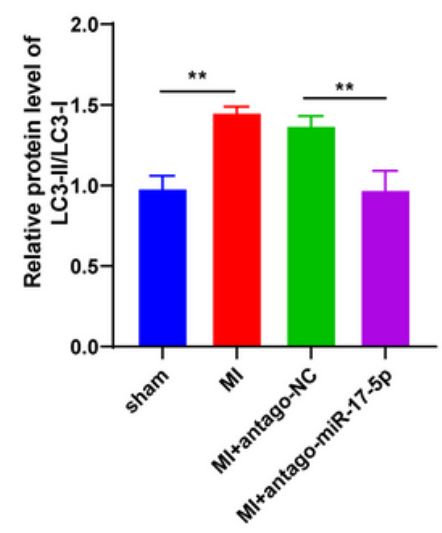

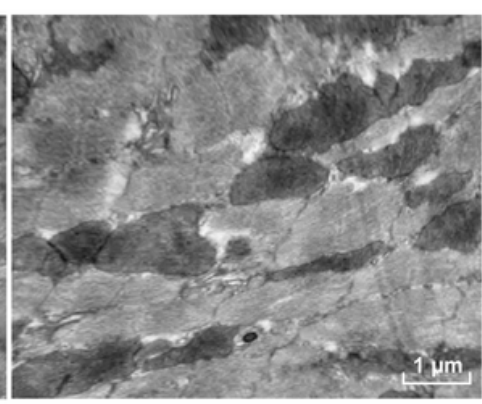

Ml+antago-NC

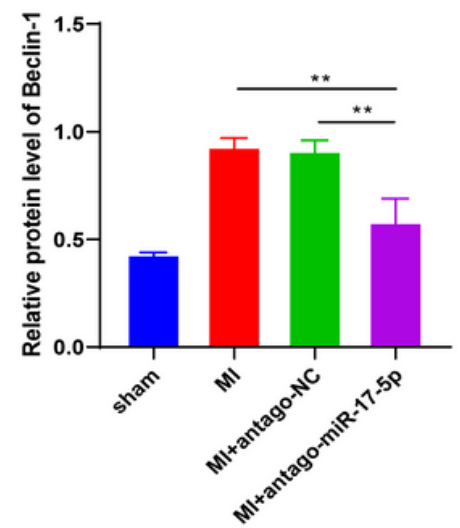

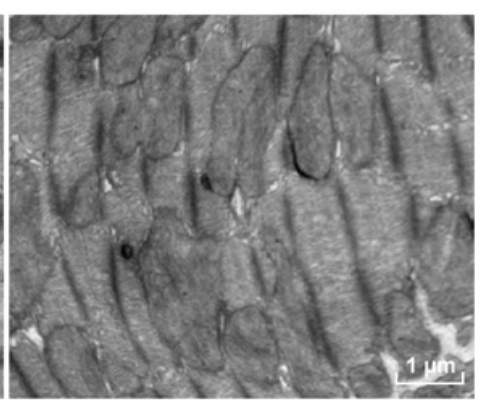

Ml+antago-miR-17-5p

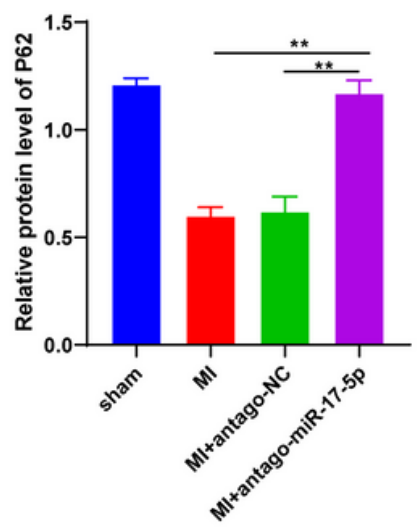

\section{Figure 3}

Inhibition of miR-17-5p expression suppressed autophagy in cardiomyocyte. A: autophagic vacuoles observed using transmission electron microscopy (scale bar, $500 \mathrm{~nm}$ ); B: the levels of autophagic markers LC3-II/LC3-I, Beclin-1 and p62 in cardiomyocyte were detected by Western blot. $\mathrm{N}=6$. The data in the figure are all measurement data, presented as mean \pm standard deviation. Data in panel $\mathrm{C}$ were analyzed using one-way ANOVA, and data in panel B were analyzed using two-way ANOVA, followed by Tukey's multiple comparisons test. ${ }^{*} \mathrm{P}<0.01$. 


\section{A}

Binding Site of hsa-miR-17-5p on STAT3:

\begin{tabular}{|c|c|c|c|c|c|c|c|}
\hline Show & $10 \quad$ entries & & & & & $\operatorname{arch}:$ & \\
\hline Bindi & ngSite & $\uparrow$ & Class $\uparrow \downarrow$ & Alignnent & $\uparrow \downarrow$ & Ag oExpllun $\downarrow$ & CleaveExpIhun $t \downarrow$ \\
\hline$\underline{\operatorname{chr} 17}$ & $: 40467309-40467315[-]$ & $\uparrow$ & $7 \operatorname{mer}-m 8 \uparrow$ & $\begin{array}{c}\text { Target: } 5^{\prime} \text { acuCCUGGCAUU----GCACUUUn } 3^{\prime} \\
|||||||:||||||||| \\
\text { miRNA : } 3^{\prime} \text { gauGGA-CGUGACAUUCGUGAAC } 5^{\prime}\end{array}$ & $\uparrow$ & 32 & 0 \\
\hline
\end{tabular}

\begin{tabular}{l|l|l} 
Previous & 1 & Next
\end{tabular}

B

miR-17-5p 3'-GGA- CGUGACAUUCGUGAAAC 5'

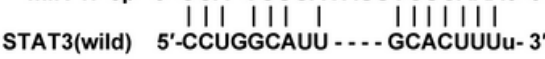
STAT3(mut) 5'-CCUGGCAUU ..... GCACTTTU- 3'
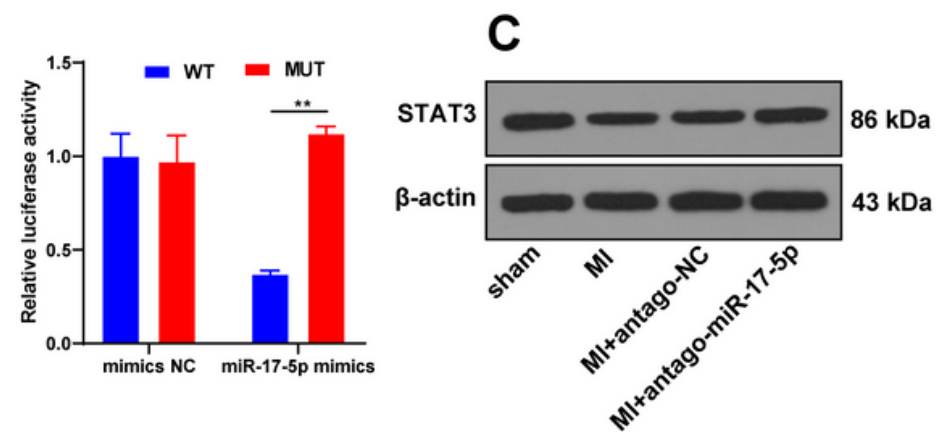

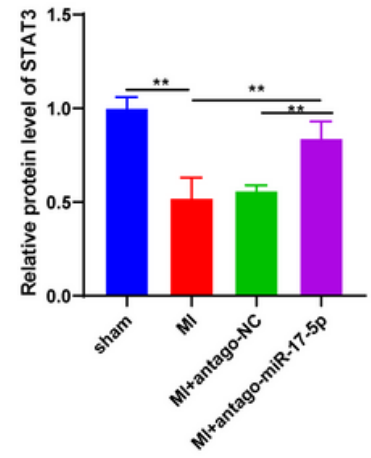

\section{Figure 4}

miR-17-5p targeted STAT3. A: the binding site of miR-17-5p on STAT3; $B$ : the relative luciferase activity of miR-17-5p mimic and mimic NC; $C$ : the regulation effect of miR-17-5p on relative expression of STAT3 detected by Western blot. $\mathrm{N}=6$. The data in the figure are all measurement data, and presented as mean \pm standard deviation. Data in panel $\mathrm{C}$ were analyzed using one-way ANOVA, and data in panel B were analyzed using two-way ANOVA, followed by Tukey's multiple comparisons test. **P $<0.01$. 

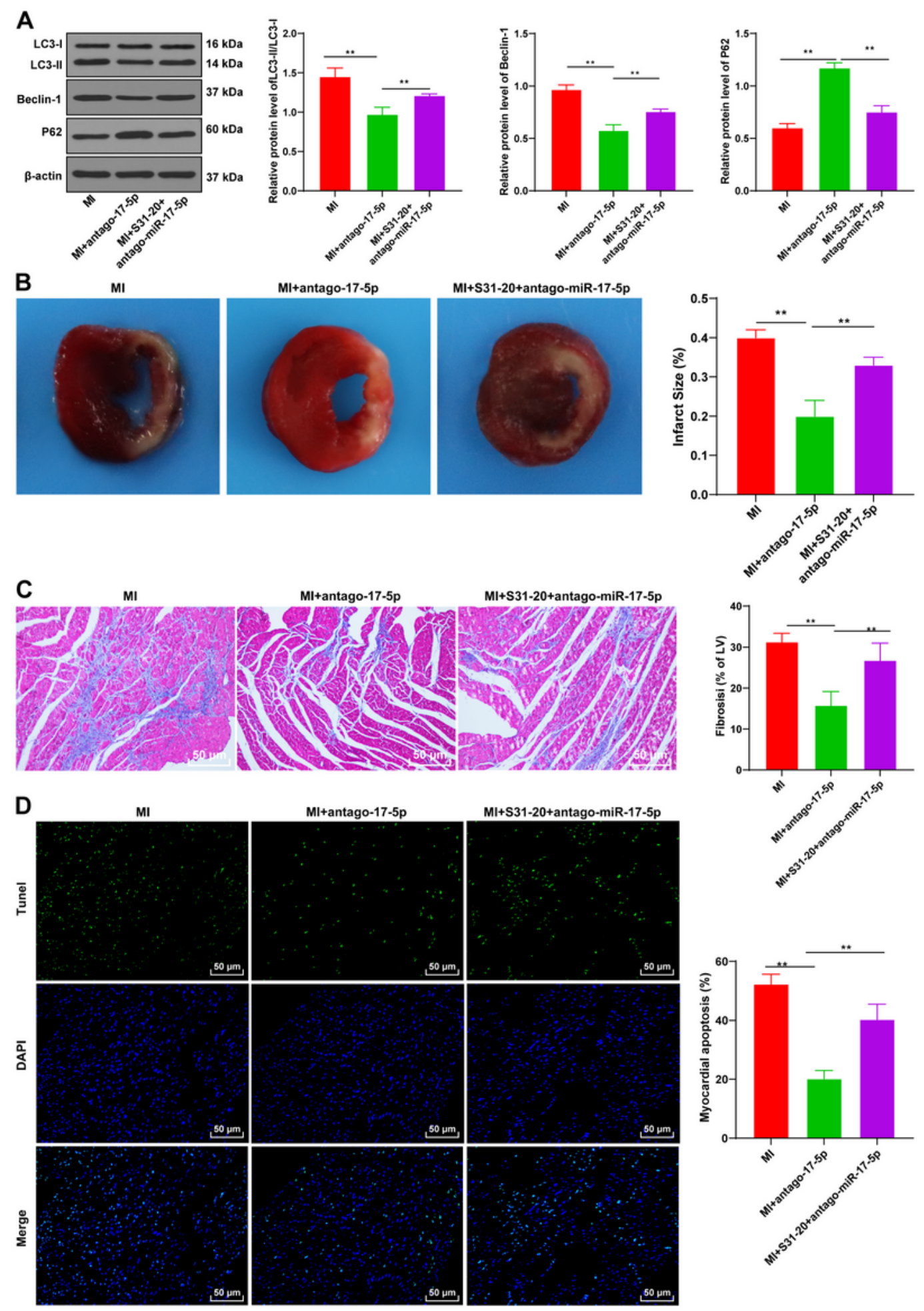

\section{Figure 5}

Inhibition of STAT3 expression reversed the improving effect of antago-miR-17-5p to MI. STAT3 inhibitor S31-20 was injected into the myocardial tissues around the ligation site of mice hearts, with mice in MI group and miR-17-5p inhibition group as controls. (A) expressions of autophagic specific markers LC3, Beclin-1 and p62 detected by Western blot; (B) infarction size was measured by TTC staining method; (C) Masson staining was used to determine myocardial fibrosis after MI (the tissues stained with blue color 
represented fibrosis) and the percentage of fibrosis area was compared among groups; (D) TUNEL staining was performed to determine cell apoptosis. $\mathrm{N}=6$. The data in the figure are all measurement data and presented as mean \pm standard deviation. Data were analyzed using one-way ANOVA, followed by Tukey's multiple comparisons test. ${ }^{* *} \mathrm{P}<0.01$.

\section{Supplementary Files}

This is a list of supplementary files associated with this preprint. Click to download.

- 3B.jpg

- 4C.jpg

- 5A.jpg 